\title{
Studying the XML Web: Gathering Statistics from an XML Sample
}

Denilson Barbosa • Laurent Mignet • Pierangelo Veltri

(C) Springer Science + Business Media, LLC 2006

The uncorrected proof of this paper was mistakenly published in volume 8 , number 4 of this journal, as well as online. The corrected version follows. This was a typesetter's error.

The online version of the original article can be found at http://dx.doi.org/10.1007/s11280-005-1544-y 